\title{
AURKB and MAPK involvement in the regulation of the early stages of mouse zygote development
}

\author{
XU Lin ${ }^{1}$, LIU Tong ${ }^{2}$, HAN Feng $^{1}$, ZONG ZhiHong ${ }^{1}$, WANG GuoLi ${ }^{1}$, YU BingZhi $^{1}$ \\ \& ZHANG Jie ${ }^{1 *}$ \\ ${ }^{1}$ Department of Biochemistry and Molecular Biology, China Medical University, Shenyang 110001, China; \\ ${ }^{2}$ Department of Gynecology and Obstetrics, Shengjing Hospital of China Medical University, Shenyang 110001, China
}

Received May 16, 2011; accepted November 15, 2011

\begin{abstract}
Aurora kinases have become a hot topic for research as they have been found to play an important role in various stages of mitotic cell division and to participate in malignant conversions of tumors. The participation of Aurora kinases in the regulation of oocyte meiosis has been recently reported, but their participation in mammalian early embryonic development remained unclear. The object of our study was to establish the spatio-temporal expression pattern of Aurora kinase B (AURKB) in mouse zygotes during the first cleavage, to reveal its functions in the early development of mouse zygotes, and to define the involvement of AURKB in mitogen-activated protein kinase (MAPK) signaling. Our results showed that in mouse zygotes AURKB expression increased in G1 phase and peaked in M phase. AURKB protein distribution was found to be in association with nuclei and distributed throughout the cytoplasm in a cell cycle-dependent manner. Functional disruption of AURKB resulted in abnormal division phenotypes or mitotic impairments. U0126, a specific mitogen-activated protein kinase kinase (MEK) inhibitor, caused significantly altered morphologies of early embryos together with a decrease in protein expression and kinase activity of AURKB. Our results indicated that the activity of AURKB was required for regulating multiple stages of mitotic progression in the early development of mouse zygotes and was correlated with the activation of the MAPK pathway.
\end{abstract}

AURKB, MAPK, mouse zygote, mitosis, cell cycle regulation

Citation: Xu L, Liu T, Han F, et al. AURKB and MAPK involvement in the regulation of the early stages of mouse zygote development. Sci China Life Sci, 2012, 55: 47-56, doi: 10.1007/s11427-012-4264-4

Aurora kinases have been implicated in several vital events in mitosis. They represent a serine/threonine kinase family highly conserved during evolution. Aurora kinases are different in their sub-cellular distribution and functions. In somatic cells, Aurora kinase B (AURKB), the "chromosomal passengers" protein, has been found at the midbody of anaphase cells and at the post-mitotic bridge of telophase cells, participating in chromatin modification, microtubule-kinetochore attachment, spindle checkpoint and cytokinesis [1]. Aurora kinases are over-expressed in a variety of tumor cell lines, suggesting that these kinases might play

*Corresponding author (email: jiezhangcmu@ @otmail.com) a role in tumorigenesis, and have already become potential targets for cancer diagnosis and therapy. Aurora kinases in reproductive germ cells have been the focus of interest for researchers in recent years, and little was known on the subject till now. Studies have shown that meiotically incompetent mouse oocytes contained transcripts for all three $A U R K$ isoforms and transcript levels remained unchanged as oocytes progressed through meiosis, with AURKA being the predominant isoform. Inhibition of Aurora kinases resulted in aberrant chromatin remodeling and significantly fewer cells progressing to meiosis II (MII). AURKA over-expression triggered abnormal amplification of centrosomes, while AURKB-over-expressed oocytes failed in 
proper chromosomal arrangement [2,3]. These studies prompted us to question how Aurora kinases would function during development after fertilization. In somatic cells, Aurora kinases have been identified as important regulators of mitotic divisions. Nevertheless, little was known about the involvement of Aurora kinases in mammalian embryonic development.

Mitogen-activated protein kinase (MAPK) cascades are highly conserved and have central roles in diverse cellular functions. Studies on progesterone-induced maturation of Xenopus oocytes indicated that over-expression of kinase Eg2, a Xenopus member of the Aurora/Ipl1 family, activated the MAP kinase pathway [4]. This study raises the possibility that Aurora kinase signals through the MAPK signaling pathway. There have been some reports indicating crosstalk between AURKA and MAPK [5,6]. As for the relationship between AURKB and MAPK, Kanda et al. [7] showed that AURKB kinase activity was involved in Ras-mediated cell transformation. Eves et al. [8] demonstrated that the loss of Raf kinase inhibitory protein (RKIP) through hyperactivation of the Raf/MEK/ERK1,2 signaling cascade regulated the mitotic checkpoint via inhibition of AURKB. However, Kosik et al. [9] argued against the direct role for Aurora kinases in the Ras/MAPK pathway: they found that AURKA activity was not a major requirement for Ras/MAPK signaling. Another report demonstrated that during oocyte maturation, active maturation-promoting factor (MPF) was necessary and sufficient to induce AURKA phosphorylation and activation, independently of the Mos/MAPK pathway [10]. In mammals, MAPK members include extracellular signal-regulated kinases 1 and 2 (ERK1/2; MAPK3 and MAPK1, respectively). There have been some observations clearly demonstrating that Mos, MAPK and MAPK substrates are all involved in regulating microtubule dynamics and the size of the first polar body of mouse oocytes [11,12]. However, there is limited information related to understanding of Aurora kinases and MAPK interaction in regulating early embryonic development in mice, which is important to further understand the mechanism of these kinases regulating cell cycle events. A previous study identified the expression of AURKA in mouse zygotes [13]. The objectives of our study were to determine the expression pattern of AURKB during the first cleavage in mouse zygotes, to examine the effects of functional disruption of AURKB during early development of mouse zygotes, and to identify the involvement of AURKB in the MAPK pathway.

\section{Materials and methods}

\subsection{Chemicals}

All the chemicals used in this study were purchased from Sigma Aldrich Corporation (USA) except for those specifically mentioned. MEK inhibitor U0126 was prepared as a stock solution dissolved in dimethyl sulfoxide (DMSO). Hyaluronidase was prepared in M2 medium directly and stored at $-20^{\circ} \mathrm{C}$.

\subsection{Mouse stimulation, fertilization and collection of zygotes}

Kunming white strain mice were used in this study. Animal care and handling were conducted in accordance with policies on the care and use of animals promulgated by the ethical committee of China Medical University. Female mice were injected with 10 IU of pregnant mare serum gonadotropin (PMSG) and then with 10 IU of human chorionic gonadotropin (hCG) $48 \mathrm{~h}$ later. In vivo-fertilized zygotes were collected $19 \mathrm{~h}$ post hCG injection from the oviduct ampullae of super-ovulated females that had mated with males of the same strain. After removing cumulus cells with $300 \mathrm{IU} \mathrm{mL} \mathrm{mL}^{-1}$ hyaluronidase in M2 medium, zygotes were cultured and kept in M16 medium at $37^{\circ} \mathrm{C}$ in a humidified atmosphere of $5 \% \mathrm{CO}_{2}$ until use. The development model of mouse one-cell embryos is as follows: G1 phase, 12-21 h post hCG injection; S phase, 21-27 h post hCG injection; G2 phase, 27-30 h post hCG injection; M phase, 30-33 h post hCG injection.

\subsection{RNA isolation, reverse transcription and real time PCR}

Zygote total mRNA was extracted from 100 zygotes at each development stage using the Illustra QuickPrep Micro mRNA Purification Kit (GE Healthcare, Boston, MA, USA) according to the manufacturer's instructions. Zygote cDNA was synthesized using $20 \mathrm{pmol} \mathrm{dT}$ primer, $0.5 \mathrm{mmol} \mathrm{L}^{-1}$ dNTP, $20 \mathrm{U}$ RNase inhibitor and $1 \mu \mathrm{L}$ ImProm-II Reverse Transcriptase (Promega, Madison, WI, USA) in a final volume of $20 \mu \mathrm{L}$. For each gene, species-specific primers crossing exon junction were designed using the sequences acquired from the GenBank database. The primers for mouse AURKB (GenBank ID: NM_011496.1) were forward primer 5'-GCCAAAGTCTGCAATCTTCA-3'; reverse primer 5'-CCTGACCTACTGCCACAAGA-3'. The following primers were used for $A C T B$ (GenBank ID: NM_007393.3): forward primer 5'-GTCTTTACGGATGTCAACG-3'; reverse primer 5'-TTTCCAGCCTTCCTTCTT-3'. Real time PCR was performed on the Applied Biosystems 7500 real time PCR system (USA). Each PCR was performed with 2.5 zygote equivalents of cDNA added to QuantiTect SYBR Green Master Mix (Qiagen, Boston, MA, USA). Real time PCR reactions were carried out for 45 cycles $\left(94^{\circ} \mathrm{C}\right.$ for $15 \mathrm{~s}$, $53^{\circ} \mathrm{C}$ for $30 \mathrm{~s}, 72^{\circ} \mathrm{C}$ for $35 \mathrm{~s}$ ) after an initial $15 \mathrm{~min}$ incubation at $95^{\circ} \mathrm{C}$. Data were collected from experiments in three replicates with triplicate samples for each gene, and fold increases were based on the metaphase of meiosis IIarrested egg levels, which were normalized to 1. Data were analyzed using comparative $\mathrm{Ct}$ (threshold cycle) method. 


\subsection{Western blot analysis}

Groups of zygotes $(n=250)$ were prepared for Western blot analysis. Zygotes were placed in $2 \times$ sodium dodecyl sulphate (SDS)-polyacrylamide gel electrophoresis (PAGE) sample loading buffer, vortexed and placed on ice for 15 min. Following sonication on ice for $10 \mathrm{~s}$, samples were denatured at $90^{\circ} \mathrm{C}$ for $10 \mathrm{~min}$ and loaded for electrophoresis. Total protein from equal numbers of mouse zygotes was loaded in each lane and separated by one-dimensional SDS-PAGE. Resolving gels were cast using $12 \%$ acrylamide, stacking gels contained 5\% acrylamide. Gels were equilibrated and transferred to PVDF transfer membrane (PALL, USA). Blots were blocked in 5\% nonfat milk in Tris-buffered saline (TBS) with $0.5 \%$ Tween (TBST) at $37^{\circ} \mathrm{C}$ for $1 \mathrm{~h}$ and incubated with the appropriate primary antibody diluted in TBST with 5\% nonfat milk overnight at $4^{\circ} \mathrm{C}$ with agitation. Antibodies included anti-AURKB (1:200, Santa Cruz, CA, USA), anti-tERK1/2 (1:1000, Cell Signaling, Beverly, MA, USA), anti-pERK1/2 antibody (1:1000, Cell Signaling, Beverly, MA, USA) and anti- $\alpha$-tubulin antibody (1:1000, Beyotime Biotechnology, Nantong, China). After washing with TBST, blots were incubated with the appropriate horseradish peroxidase (HRP)-conjugated IgG secondary antibody (1:4000, Santa Cruz, CA, USA) at room temperature for $1 \mathrm{~h}$, washed in TBST and developed with ECL Plus reagents (Beyotime Biotechnology, Nantong, China) according to the manufacturer's instructions. Band densities were assessed using NIH imaging software, Image J.

\subsection{Plasmid construction and microinjection}

We used the mammalian expression vector pGPU6/GFP/ Neo (GenePharma, Shanghai, China) to direct the synthesis of small interfering RNA (siRNA)-like transcript for suppression of $A U R K B$ expression. Two 64-mer oligos for $A U R K B$ short hairpin RNA (shRNA), one forward 5'-CACCGCCAGAAGTTGGTCGAGAACATTCAAGAG ATGTTCTCAGCCAACTTCTGGCTTTTTTG-3', one reverse 5'-GATCCAAAAAACCAGAATTTGGCTGAGAACATCTCTTGAATGTTCTCAGCCAACTTCTGGC-3', were synthesized (GenePharma, Shanghai, China), annealed, phosphorylated and ligated into the $B b s$ I and BamH I restriction sites of the digested pGPU6/GFP/Neo vector. The recombinant plasmid was sequenced to verify the correct insert. The plasmid was diluted in endotoxin-free TE buffer $(\mathrm{pH} 7.4)$ at different concentrations and microinjected into zygotes in G1 phase using the TransferMan microinjection system (Eppendorf, Germany) and a model IX-70 inverted microscope (Olympus, Japan) with Hoffmann optics. Typical injection volume was $5 \%$ of the total cell volume or 10 pL per zygote. Green fluorescent protein (GFP) expression was examined $24 \mathrm{~h}$ after microinjection using a fluorescence microscope (Olympus, Japan). Zygotes expressing
GFP were collected for further experiments. Zygotes injected with TE buffer or scrambled shRNA served as negative controls.

\subsection{Zygote culture and MEK inhibition}

MEK inhibitor U0126 was dissolved in DMSO to obtain a $10 \mathrm{mmol} \mathrm{L}^{-1}$ stock. Stock solution was dissolved in $\mathrm{M} 2$ medium to obtain final concentrations of $10,20,30 \mu \mathrm{mol}$ $\mathrm{L}^{-1}$. Control treatments contained $0.1 \%$ DMSO. To assess the effects of MEK inhibition on zygotic development, zygotes in G1 phase were cultured in the presence or absence of various doses of U0126. Zygotes were assessed by Western blot analysis to determine the optimal inhibition concentration at $33 \mathrm{~h}$ post hCG injection. To determine the effects of ERK1/2 inactivation, G1 zygotes were incubated in vitro until $\mathrm{M}$ phase in the presence or absence of U0126 and then subjected to immunofluorescence staining and confocal microscopy or processed for morphology detection by phase contrast microscopy (Nikon, Japan).

\subsection{Immunofluorescence}

To investigate the spatial pattern of AURKB, zygotes were fixed in $4 \%$ paraformaldehyde in phosphate buffered saline (PBS) ( $\mathrm{pH} \mathrm{7.4)} \mathrm{for} \mathrm{at} \mathrm{least} 30 \mathrm{~min}$ at room temperature. After being permeabilized with $3 \%$ Triton $\mathrm{X}-100$ at $37^{\circ} \mathrm{C}$ for $15 \mathrm{~min}$, zygotes were blocked in $2 \%$ bovine serum albumin (BSA) at $37^{\circ} \mathrm{C}$ for $30 \mathrm{~min}$ and incubated at $4^{\circ} \mathrm{C}$ overnight with goat anti-AURKB (1:100, Santa Cruz, CA, USA). After three washes with the blocking solution, the appropriate Alexa 594 conjugated secondary antibodies (Millipore, Bedford, MA, USA) were added at a 1:500 dilution for $1 \mathrm{~h}$ at $37^{\circ} \mathrm{C}$. Following washing, slides were incubated with $4 \mathrm{mg} \mathrm{mL}^{-1}$ Hoechst 33342 in PBS for $5 \mathrm{~min}$ at $37^{\circ} \mathrm{C}$. For double-staining of AURKB and $\alpha$-tubulin, goat anti-AURKB antibody and mouse anti- $\alpha$-tubulin antibody (1:500, Beyotime Biotechnology, Nantong, China) were added together and the secondary antibodies used were Alexa 594 and FITC-conjugated, respectively. For double-staining of pERK1/2 and AURKB, the above method was employed, except that one of the primary antibodies used was 1:500 rabbit anti-pERK1/2 antibody. Coverslips were then mounted on glass slides with $90 \%$ glycerol in PBS for fluorescence microscopic visualization under a laser scanning confocal microscope at $\times 400$ magnification (Olympus, Japan).

\subsection{AURKB activity assay}

Zygotes were collected, 15 per group, and lysed at $4{ }^{\circ} \mathrm{C}$ in 4 volumes of $\mathrm{EB}$ buffer $\left(80 \mathrm{mmol} \mathrm{\textrm {L } ^ { - 1 }} \beta\right.$-glycerophosphate, $\mathrm{pH}$ 7.3, $20 \mathrm{mmol} \mathrm{L}{ }^{-1}$ EGTA, $15 \mathrm{mmol} \mathrm{L}{ }^{-1} \mathrm{MgCl}_{2}, 1 \mathrm{mmol}$ $\mathrm{L}^{-1}$ dithiothreitol), supplemented with protease inhibitor mixture and $1 \mu \mathrm{mol} \mathrm{L}{ }^{-1}$ okadaic acid. Lysates were centri- 
fuged at $15000 \times g$ at $4^{\circ} \mathrm{C}$ for $15 \mathrm{~min}$ and then immunoprecipitated with anti-AURKB antibody. Histone H3 kinase activity of AURKB was assayed in the corresponding immunoprecipitates, incubated for $30 \mathrm{~min}$ at $30^{\circ} \mathrm{C}$ in the presence of kinase buffer $\left(50 \mathrm{mmol} \mathrm{L}^{-1}\right.$ Tris- $\mathrm{HCl}, \mathrm{pH} 7.2,15$ mmol L ${ }^{-1} \mathrm{MgCl}_{2}$, 5 mmol L ${ }^{-1}$ EGTA, 1 mmol L ${ }^{-1}$ dithiothreitol) containing $3 \mu \mathrm{Ci}$ of $\left[\gamma^{-}{ }^{32} \mathrm{P}\right]$ ATP (Beijing FuRui Biotechnology, Beijing, China), $50 \mu \mathrm{mol} \mathrm{L}^{-1}$ ATP and 0.5 $\mathrm{mg} \mathrm{mL}^{-1}$ of histone $\mathrm{H} 3$. Kinase reaction was stopped by adding Laemmli buffer and boiling. After electrophoresis and autoradiography, the band corresponding to histone $\mathrm{H} 3$ was excised, and the associated radioactivity was measured in a liquid scintillation counter (Beckman Coulter, USA).

\subsection{Statistical analysis}

All experiments were performed independently at least three times. Student $t$-test was used to evaluate the differences between multiple experimental groups using SPSS 12.0 software, and the differences were considered statistically significant at $P<0.01$.

\section{Results}

\subsection{The expression of AURKB in mouse zygotes}

To determine the $A U R K B$ isoform present in mouse oocytes, an isoform-specific primer was designed for $A U R K B$ and used in real time PCR for transcript amplification. The results revealed that $A U R K B$ was increasingly expressed from phase G1 and peaked at phase M (Figure 1A). Accordingly, the Western blot analysis indicated a similar protein expression pattern. Polyclonal goat antibody to AURKB revealed a single protein at approximately $39 \mathrm{kD}$ in an extract prepared from 250 zygotes (Figure 1B). Thus, AURKB was expressed at four phases during the first cleavage in mouse zygotes, with the amount reaching maximum after the commencement of the mitotic process, both at the mRNA and protein levels.

\subsection{Localization of AURKB at different stages during the first cleavage}

AURKB localization in zygotes during the first cleavage was detected using indirect immunofluorescence (Figure 2). In G1 and S phase zygotes, it was mainly present in the subcortical region and slightly in the cytoplasm. In G2 zygotes, pronuclei broke down and visible nucleoli disappeared. The embryo surface was ruffling and the cytoskeleton was reorganized preparatory to cleavage. In this phase, AURKB was not only dispersed throughout the cytoplasm, but also showed accumulation near chromatin. With the entrance into anaphase of mitosis, AURKB was expressed uniformly throughout the cell.
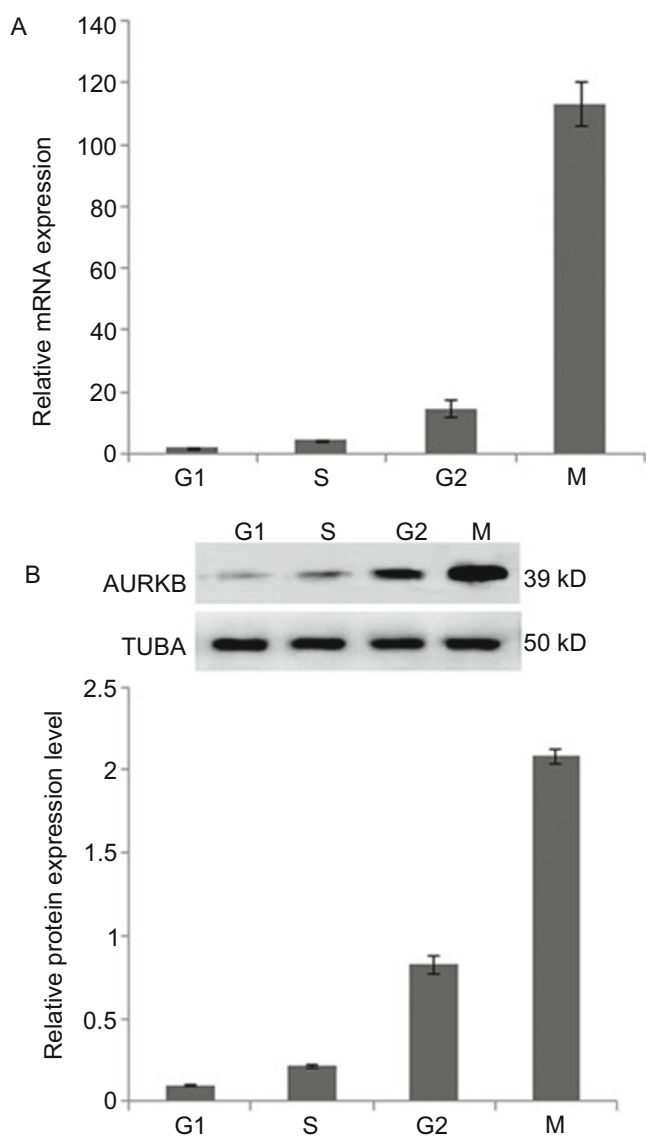

Figure 1 The expression pattern of AURKB at four phases during the first division of mouse zygotes. A, Graphical representation demonstrating fold-increases of $A U R K B$ mRNA levels between various zygote types obtained from real time PCR. Oocytes arrested in metaphase of meiosis II (Met II) were used as the control and were normalized to 1 (not shown). B, Detection of mouse AURKB protein by western blotting. $\alpha$-tubulin was used as a loading control.

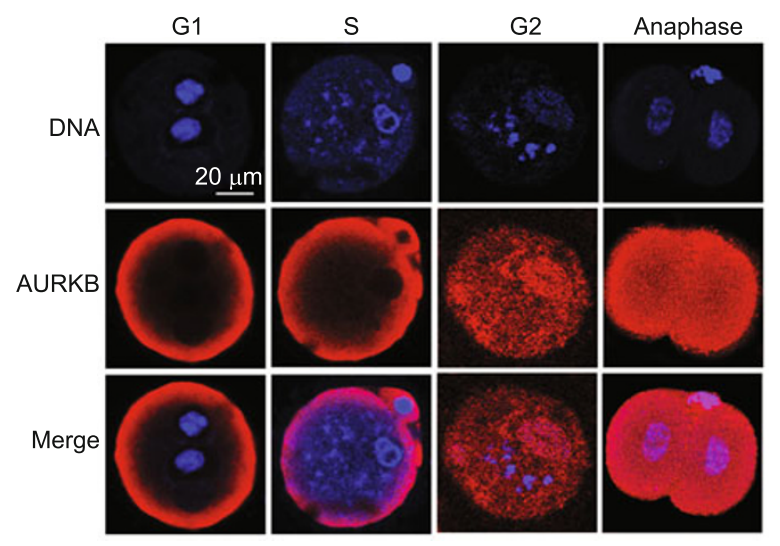

Figure 2 Localization of AURKB during the process of the first cleavage. Red, AURKB; blue, chromatin; merged, overlapping of red and blue.

\subsection{AURKB-knockdown zygotes failed in proper chromosome segregation and cytokinesis}

We used RNAi to assess loss-of-function of AURKB during 
the first mitotic process. Correct recombination of the shRNA expression plasmid for $A U R K B$ was confirmed by sequencing. The plasmid was then injected into the male pronuclei of G1 zygotes at concentrations of $0.2,0.4,0.6$, $0.8,1.0$ and $1.2 \mathrm{mg} \mathrm{mL}^{-1}$, and the zygotes were then cultured in M16 medium for $24 \mathrm{~h}$ to allow time for RNAi-mediated targeting of mRNA, which was assessed by real time PCR and Western blotting. The experiments showed that $1.0 \mathrm{mg} \mathrm{mL}^{-1}$ was a suitable concentration that resulted in the strongest suppression without embryo lethality caused by over-microinjection. As expected, the level of $A U R K B$ mRNA decreased significantly $(P<0.01)$ (Figure $3 \mathrm{~A})$. Moreover, a significant difference $(P<0.01)$ in protein expression was observed between RNAi groups and control groups when analyzed by Western blotting (Figure 3B). We then examined the cleavage results after functional disruption of AURKB. Compared to the two controls, zygotes of the $A U R K B$-knockdown group were $50 \%$ more likely to have abnormal cleavage and asymmetrical cytokinesis (Figure $3 \mathrm{C}$ and D).

To further characterize AURKB-RNAi effect, embryos were fixed and stained with Hoechst 33342 and antibodies specific for AURKB and $\alpha$-tubulin. Immunofluorescence staining revealed that a significantly larger percentage of embryos developed to three or more unequal-sized daughter cells. AURKB protein staining was nearly absent in each embryo (Figure 3E). These results suggest that although many zygotes were able to enter cleavage, they were undergoing irregular divisions.

\subsection{The effect of ERK1/2 activity inhibition on the cleavage condition in mouse zygotes}

We ascertained the presence of ERK $1 / 2$ by Western blot analysis at four phases during the first division of mouse zygotes. ERK1/2 protein levels increased from G1 phase and peaked at $M$ phase. Phospho-ERK1/2 antibody was added to confirm that ERK1/2 achieved its highest activity during $M$ phase (Figure 4).

Next, we used U0126, a specific inhibitor of MEK, to test whether down-regulation of the MAPK pathway affected the cleavage condition of mouse zygotes. U0126 at concentrations of 10,20 and $30 \mu \mathrm{mol} \mathrm{L} \mathrm{L}^{-1}$ was used to determine the appropriate working concentration. As shown in Figure 5A, $30 \mu \mathrm{mol} \mathrm{L}{ }^{-1} \mathrm{U} 0126$ was able to suppress the phosphorylation level of ERK1/2 significantly to below $10 \%$ of that of control. We then proceeded with our experiments using that concentration. As shown in Figure 5B, altered morphologies were observed after the treatment. Nearly $80 \%$ of U0126-treated embryos showed abnormal or incomplete cytokinesis, and formed two or three daughter cells of different sizes or with altered shapes (Figure 5C). Almost all zygotes divided, although with clearly altered morphologies, and ultimately died before reaching the morula stage (not shown).

\subsection{AURKB was down-regulated by ERK inactivation}

To determine the effect of ERK inactivation on AURKB, zygotes treated with $30 \mu \mathrm{mol} \mathrm{L}{ }^{-1}$ U0126 were collected for Western blotting and kinase activity assay. Since U0126 was used in a final concentration of $0.1 \%$ DMSO, we set another control group containing $0.1 \%$ DMSO. We found that AURKB protein was down-regulated both in expression levels and kinase activity. As shown in Figure 6A, AURKB protein was reduced by $68.77 \%$ compared to the DMSO-treated control, with no significant differences between the two controls. Next, we analyzed whether the MEK inhibitor affected AURKB activity. We observed that the activity decreased by $65.86 \%$ in response to ERK $1 / 2$ phosphorylation abortion (Figure 6B). These results prompted us to investigate whether U0126 altered the co-localization of AURKB and phospho-ERK1/2. Control embryos were highly fluorescent throughout the cytoplasm when double-stained with phospho-ERK1/2 and AURKB antibodies, which suggested they shared a similar localization pattern. We observed that in the treated group some zygotes normally formed a contractile ring between two sets of chromosomes but failed to divide normally into two identical daughter cells of the same size (Figure 6C(b)). Several furrows were also observed in a number of treated eggs that formed three sets of chromosomes. Those zygotes with multiple furrows seemed to form tripolar embryos (Figure 6C(c)). Meanwhile, after the $30 \mu \mathrm{mol} \mathrm{L}{ }^{-1} \mathrm{U} 0126$ treatment, activated ERK1/2 protein became much less detectable, especially in and around newly formed nuclei (Figure $6 \mathrm{C}\left(\mathrm{b}_{2}\right)$ and $\left(\mathrm{c}_{2}\right)$ ). The anti-AURKB signal was also highly reduced, similar to the change of phospho-ERK1/2 (Figure $6 \mathrm{C}\left(\mathrm{b}_{3}\right)$ and $\left(\mathrm{c}_{3}\right)$ ), and some fluorescence was visible at the membrane (Figure $6 \mathrm{C}\left(\mathrm{c}_{3}\right)$ ).

\section{Discussion}

In this study, we determined the expression pattern of AURKB during the first cleavage of early mouse embryos. The expression level was low in G1/S but high in G2/M and peaked at M. This showed a cell cycle-dependent change in the expression, similar to that in somatic cells. Mouse or bovine oocytes also contain a transcript for AURKB and the transcript level increases significantly as oocytes gain meiotic competence, but does not change as oocytes progress through meiosis to MII $[14,15]$. We could speculate that AURKB might contribute to mouse zygotic development at different steps throughout the process of the first mitosis at different expression levels. We also analyzed the intracellular localization of AURKB at four phases throughout the first cleavage of mouse zygotes. With lower protein levels at G1/S, it was present throughout the cytoplasm and hardly detectable around male and female pronuclei. At G2 phase, the fluorescence intensity of AURKB increased in the 

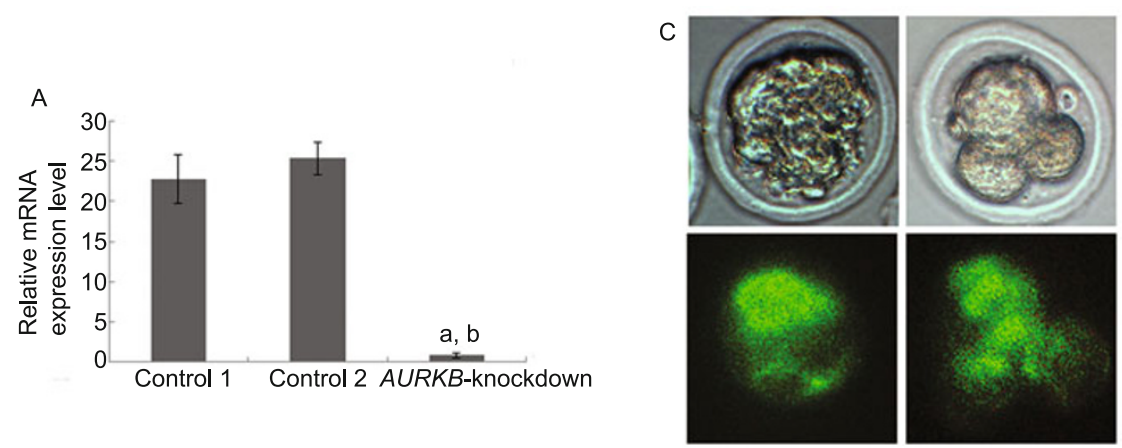

$50 \mu \mathrm{m}$

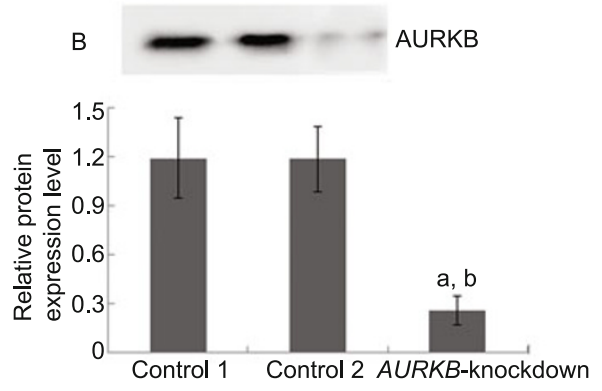

D

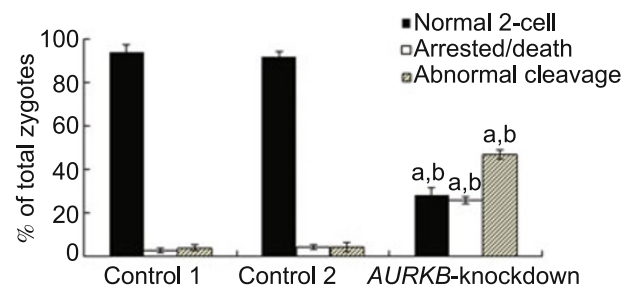

$\mathrm{E}$

Control

AURKB-knockdown
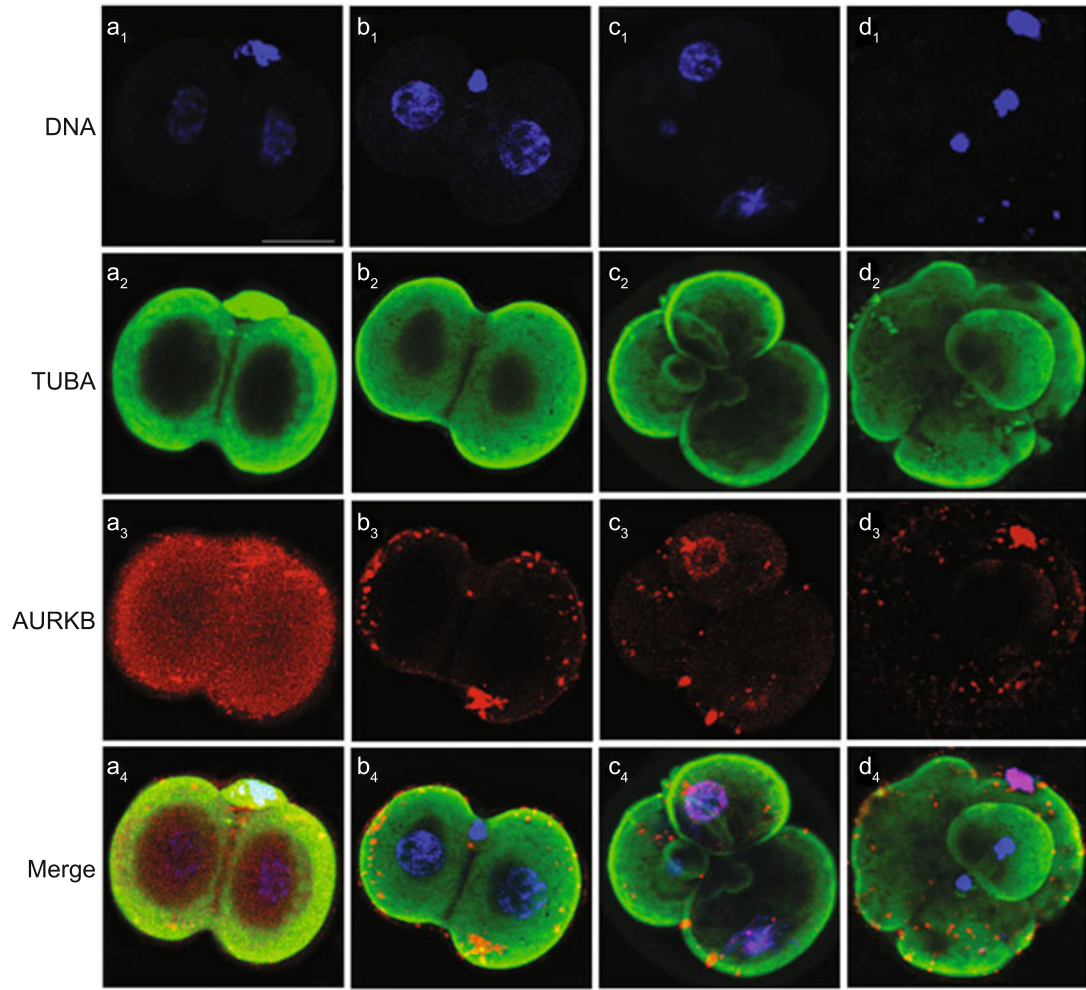

Figure 3 The effect of the microinjection of hairpin siRNA expression plasmid for AURKB. A, AURKB mRNA level in relative arbitrary units after RNAi-mediated knockdown. B, Western blot analysis to verify the suppression effect. C, Observation of typical abnormal cleavage of zygotes injected with shRNA expression plasmid for AURKB and detection of GFP expression by a fluorescence microscope. D, A bar graph representation of the average ratios of normal mitotic cells, arrested/dead cells and those with abnormal cleavage. Zygotes microinjected with the equal volume of endotoxin-free TE buffer and equal amount of scrambled shRNA served as control 1 and control 2, respectively. Letters a and b indicate significant differences compared to control 1 and control 2, respectively $(P<0.01)$. E, Fluorescence microscopy images of the effects of AURKB deficiency. Red, AURKB; green, $\alpha$-tubulin; blue, DNA; merged, overlapping of red, green and blue. 

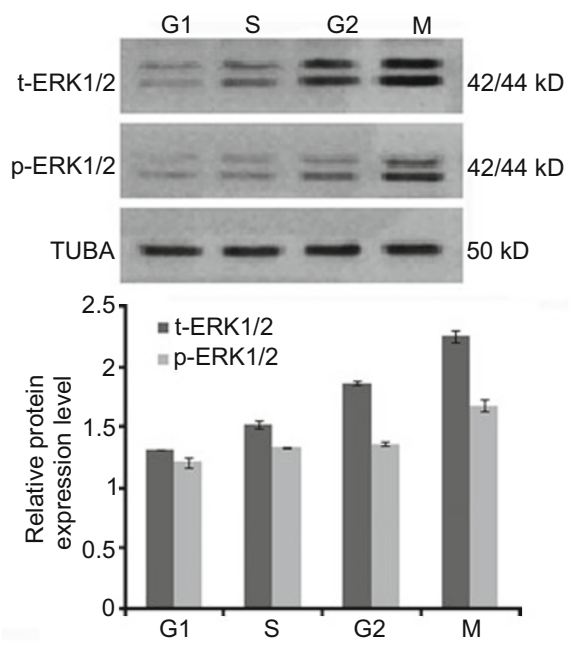

Figure 4 ERK1/2 protein expression at four phases of the first division in mouse zygotes. Graphical representation demonstrates relative protein expression levels during various zygote phases. whole cell and appeared more concentrated and associated with the chromosomes. After the zygotes entered mitosis, most of AURKB diffused throughout the cytoplasm. The success of mitosis depends to a large degree on the interaction between chromosomes and spindle microtubules, and the chromosomal passenger complex can coordinate their functions. As a member of the chromosomal passenger complex, AURKB has its characteristic localization and functions. During G2 phase, the protein clearly accumulated around the chromosome where it was regulated by other members of the passenger protein complex and participated in chromosome condensation. Phosphorylation of histone $\mathrm{H} 3$ at serine-10 during late G2/prophase by AURKB has been linked to chromosome condensation, because mutation of this phosphorylation site caused chromosomal condensation and subsequent segregation defects [16,17]. As the zygotes progressed through mitosis from metaphase to anaphase, the expression of AURKB reached its highest level,
A

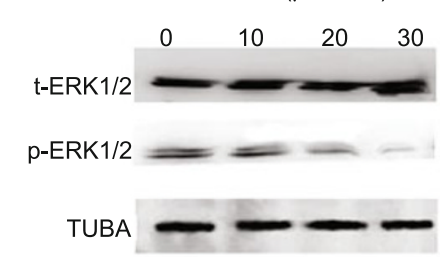

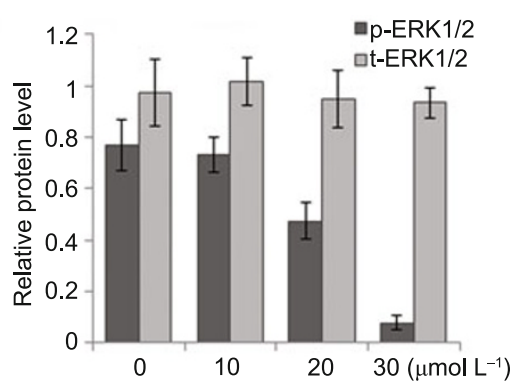
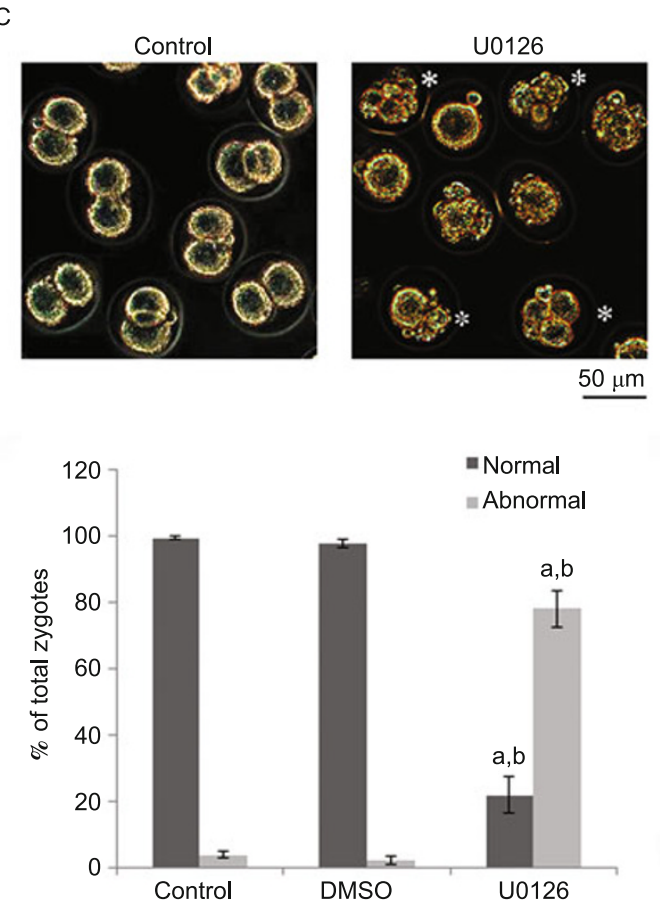

Figure 5 Effects of ERK1/2 inactivation on the cleavage condition of mouse zygotes. A, Western blotting was performed to determine the working concentration of U0126. B, Zygotes in G1 phase were treated with $30 \mu \mathrm{mol} \mathrm{L}{ }^{-1} \mathrm{U} 0126$ and observed by phase contrast microscopy $33 \mathrm{~h}$ post hCG injection. C, U0126-treated zygotes showing altered shapes were marked with a star. The bar graph represents the average ratios of normal mitotic cells and those with abnormal division. Letters $\mathrm{a}$ and $\mathrm{b}$ indicate significant differences compared to the non-treated control and $0.1 \%$ DMSO-treated control, respectively $(P<0.01)$. 

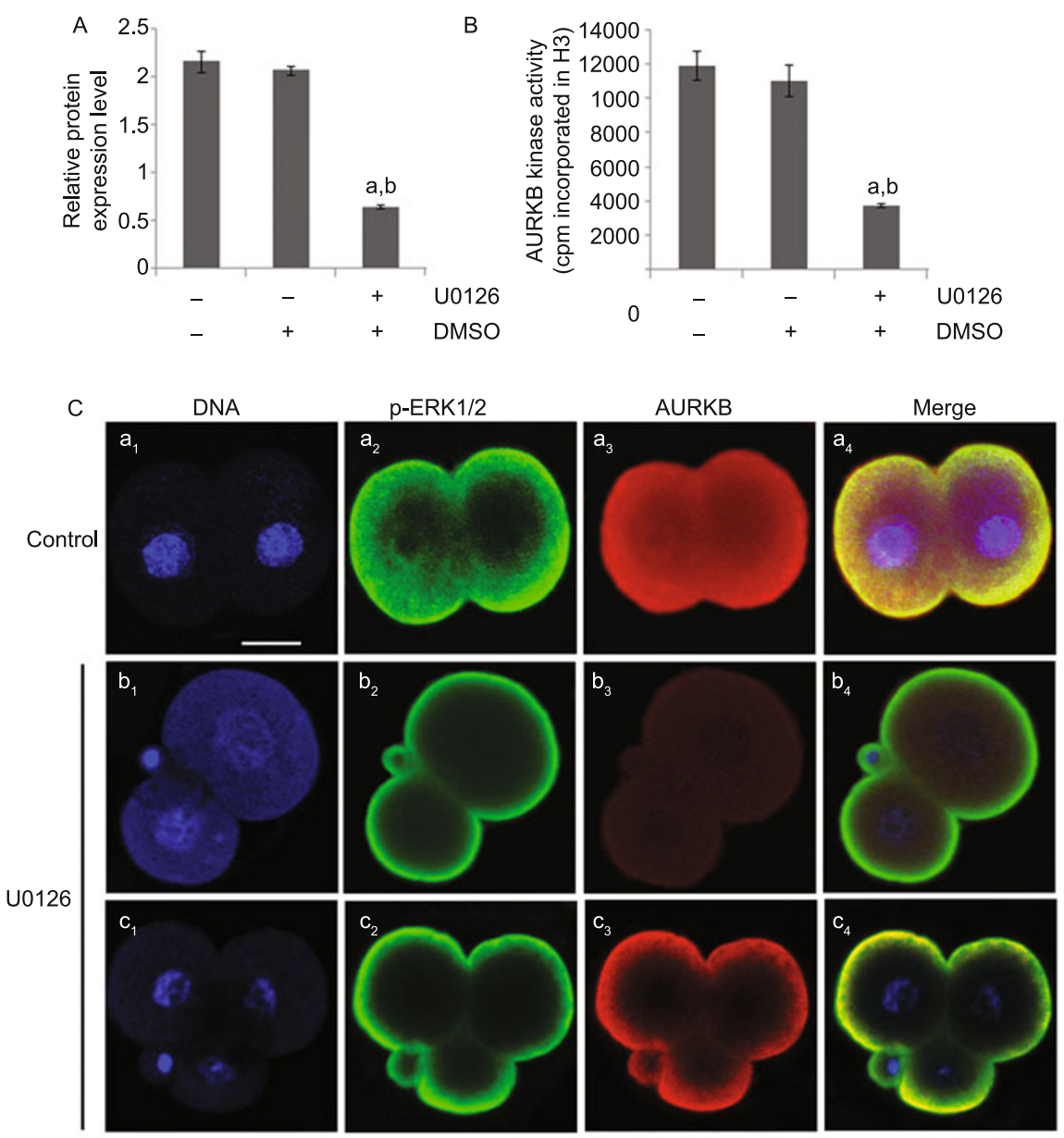

Figure 6 Effects of ERK1/2 inactivation on AURKB. A, Changes in protein expression of AURKB were detected by Western blotting after U0126 treatment. B, Protein lysates from U0126-treated embryos were immunoprecipitated with anti-AURKB antibody and assayed for AURKB kinase activity by scintillation counting and autoradiography using histone $\mathrm{H} 3$ as the substrate. The activity was expressed as cpm incorporated in the substrate. Letters a and $\mathrm{b}$ indicate significant differences compared to the no treatment group and the $0.1 \%$ DMSO group, respectively $(P<0.01)$. C, The co-localization of activated ERK1/2 and AURKB with or without U0126 treatment. Examples of alterations in U0126-treated embryos observed by confocal imaging are shown in $b_{1}-b_{4}$ and $c_{1}-c_{4}$. Red, AURKB; green, p-ERK1/2; blue, DNA; merged, overlapping of red, green and blue.

indicating its significance in cytokinesis. In mouse zygotes, from anaphase onwards, AURKB was distributed throughout the whole cell. Previous studies suggested that depletion of AURKB resulted in polyploidy due to a failure in cytokinesis through the phosphorylation of several other proteins involved in cytokinesis, although the mechanism has not yet been elucidated [18].

Since fewer studies have focused on AURKB in the regulation of oocyte or embryonic development, in this report RNA interference was used to assess the functional effect of AURKB disruption in mouse zygotes. According to a previous report, AURKB was responsible for regulating chromosome dynamics during meiosis in mouse oocytes. AURKB inhibition resulted in defects in meiotic progression and chromosome alignment at both metaphase of meiosis I (Met I) and metaphase of meiosis II (Met II), while over-expression of AURKB reversed the chromosome alignment defect, suggesting that AURKB is the primary Aurora kinase responsible for regulating chromosome dy- namics during meiosis in mouse oocytes [3]. Our results indicate that AURKB was required for the development of mouse zygotes. In our experiments, disruption of AURKB resulted in zygote lethality without completion of cytokinesis; in addition, we found that most of the AURKB-deficient mouse zygotes failed to divide normally into two identical daughter cells. Cytokinesis in some of them was random, resulting in multiple daughter cells of different sizes. During cytokinesis it is important that the cleavage furrow is positioned correctly between the two daughter nuclei after chromosome segregation. Several studies have revealed that AURKB controlled the cleavage furrow-specific Vimentin phosphorylation as well as other proteins involved in cytokinesis, such as Myosin II regulatory light chain and glial fibrillary astrocytic protein (GFAP) [19,20]. In the current study, loss of function of AURKB by RNAi caused improper positioning of the cleavage furrows followed by unequal cell division in some of the early embryos; it would also compromise the spindle checkpoint because its activity 
is required for checkpoint protein recruitment [19], which might provide a good explanation for why down-regulation of AURKB generated multinuclear phenotype and asymmetric division.

It is widely accepted that an extracellular signal regulated kinase (ERK) cascade acts at different steps during maturation and regulates meiosis in oocytes of various species [21]. A recent study in sea urchin embryos also showed that a MEK/ERK-like cascade was transiently activated at the time of mitosis [22]. Mos was once thought to play a role in regulating mitotic metaphase arrest of early mammalian embryos; however, according to the study of Kashima et al. [23], the activation of MAPK and ribosomal protein S6 kinase (Rsk) did not induce mitotic arrest in early mouse embryos. We reported here that ERK1/2 was present and highly phosphorylated at $\mathrm{G} 2 / \mathrm{M}$ phases during the first mitosis of mouse zygotes, and showed that the ERK1/2 function was required for the proper development of mouse zygotes. A study in somatic cells treated with MEK inhibitors reported mitotic defects, such as poorly defined mitotic axis and chromosome separation, suggesting that an ERK cascade acts at different levels of mitotic spindle formation [24]. MEK inhibitors could induce spindle poles to split and then lead to the formation of tri- or tetrapolar spindles under these conditions. This could be why we frequently observed formation of two daughter cells of different sizes in zygotes treated with MEK inhibitors. In the study of sea urchin embryos, ERK cascade was shown to be a part of a control mechanism that regulates mitotic spindle formation and the attachment of chromosomes to the spindle during the first mitosis [22]. Another example is that immunodepletion of MAPK in cycling Xenopus extracts or treatment with PD98059 caused termination of mitosis and mitotic microtubules abnormality followed by an unequal segregation of sister chromatids [25]. Interestingly, in our study, protein expression patterns of total and activated ERK1/2 were both similar to that of AURKB: low at G1/S phases and peaking at $M$ phase. In addition, the inactivation of ERK1/2 resulted in decreases of the AURKB protein levels and kinase activity. Irregular division was detected in the AURKB-knockdown group and the MAPK inactivation group. Similar to our findings, another study demonstrated that AURKA, along with other kinetochore-related proteins, was down-regulated by inactivation through the MAPK pathway in pancreatic cancer [26]. Another report concluded that c-Jun N-terminal kinase (JNK) signaling promoted entry into mitosis by regulating activation and expression of AURKB [27]. Together with our findings, these observations could imply some degree of crosstalk between AURKB and the MAPK signaling pathway.

In conclusion, we report that Aurora kinase B is required for the early development of mouse zygotes; we also reveal a possibility of AURKB involvement in the MAPK pathway. Future studies should focus on further exploring the mechanism and determining the targets of Aurora kinase B under- lying the regulatory function in mammalian early embryonic development.

This work was supported by the National Natural Science Foundation of China (Grant No. 81070527).

1 Fu J Y, Bian M, Jiang Q, et al. Roles of Aurora kinases in mitosis and tumorigenesis. Mol Cancer Res, 2007, 5: 1-10

2 Saskova A, Solc P, Baran V, et al. AURKA controls meiosis I progression in mouse oocytes. Cell Cycle, 2008, 7: 2368-2376

3 Shuda K, Schindler K, Ma J, et al. AURKB modulates chromosome alignment. Mol Reprod Dev, 2009, 76: 1094-1095

4 Andresson T, Ruderman J V. The kinase Eg2 is a component of the Xenopus oocyte progesterone-activated signaling pathway. EMBO J, 1998, 17: 5627-5637

5 Wan X B, Long Z J, Yan M. Inhibition of Aurora-A suppresses epithelial-mesenchymal transition and invasion by downregulating MAPK in nasopharyngeal carcinoma cells. Carcinogenesis, 2008, 29: 1930-1937

6 Tseng Y S, Lee J C, Huang F. Aurora-A over-expression enhances cell-aggregation of Ha-Ras transformants through the MEK/ERK signaling pathway. BMC Cancer, 2009, 9: 435

7 Kanda A, Kawai H, Suto S, et al. Aurora-B/AIM-1 kinase activity is involved in Ras-mediated cell transformation. Oncogen, 2005, 24: 7266-7272

8 Eves E M, Shapiro P, Naik K, et al. Raf kinase inhibitory protein regulates Aurora B kinase and the spindle checkpoint. Mol Cell, 2006, 23: $561-574$

9 Kosik A, Bekier M E, Katusin J D. Investigating the role of Aurora kinases in RAS signaling. J Cell Biochem, 2009, 106: 33-41

10 Maton G, Thibier C, Castro A, et al. Cdc2-cyclin B triggers H3 kinase activation of Aurora-A in Xenopus oocytes. J Biol Chem, 2003, 278: 21439-21449

11 Yu L Z, Xiong B, Gao W X, et al. MEK1/2 regulates microtubule organization, spindle pole tethering and asymmetric division during mouse oocyte meiotic maturation. Cell Cycle, 2007, 6: 330-338

12 Terret M E, Lefebvre C, Djiane A, et al. DOC1R: a MAP kinase substrate that control microtubule organization of metaphase II mouse oocytes. Development, 2003, 130: 5169-5177

13 Yao L J, Zhong Z S, Zhang L S, et al. Aurora-A is a critical regulator of microtubule assembly and nuclear activity in mouse oocytes, fertilized eggs, and early embryos. Biol Reprod, 2004, 70: 1392-1399

14 Swain J E, Ding J, Wu J, et al. Regulation of spindle and chromatin dynamics during early and late stages of oocyte maturation by aurora kinases. Mol Hum Reprod, 2008, 14: 291-299

15 Uzbekova S, Arlot-Bonnemains Y, Dupont J, et al. Spatio-temporal expression patterns of Aurora kinases A, B, and C and cytoplasmic polyadenylation-element protein in bovine oocytes during meiotic maturation. Biol Reprod, 2008, 78: 218-233

16 Mellone B G, Ball L, Suka N, et al. Centromere silencing and function in fission yeast is governed by the amino terminus of histone $\mathrm{H} 3$. Curr Biol, 2003, 13: 1748-1757

17 Zhang X, Lan W, Ems-McClung S C, et al. Aurora B phosphorylates multiple sites on mitotic centromere-associated kinesin to spatially and temporally regulate its function. Mol Biol Cell, 2007, 18: 3264-3276

18 Murata-Hori M, Fumoto K, Fukuta Y, et al. Myosin II regulatory light chain as a novel substrate for AIM-1, an aurora/Ipl1p-related kinase from rat. J Biochem, 2000, 128: 903-907

19 Goto H, Yasui Y, Kawajiri A, et al. Aurora-B regulates the cleavage furrow-specific vimentin phosphorylation in the cytokinetic process. J Biol Chem, 2003, 278: 8526-8530

20 Vader G, Medema R H, Lens S M. The chromosomal passenger complex: guiding Aurora-B through mitosis. J Cell Biol, 2006, 173: 833-837 
21 Abrieu A, Doree M, Fisher D. The interplay between cyclin-B-Cdc2 kinase (MPF) and MAPK during maturation of oocytes. J Cell Sci, 2001, 114: 257-267

22 Zhang W L, Huitorel P, Glass R, et al. A MAPK pathway is involved in the control of mitosis after fertilization of the sea urchin egg. Dev Biol, 2005, 282: 192-206

23 Kashima K, Kano K, Naito K. Mos and mitogen-activated protein kinase do not show cytostatic factor activity in early mouse embryos. J Reprod Dev, 2007, 53: 1175-1812

24 Willard F S, Crouch M F. MEK, ERK, and p90RSK are present on mitotic tubulin in Swiss 3T3 cells: a role for the MAP kinase pathway in regulating mitotic exit. Cell Signal, 2001, 13: 653-664

25 Yue J, Ferrell J E Jr. Mos mediates the mitotic activation of p42 MAPK in Xenopus egg extracts. Curr Biol, 2004, 14: 1581-1586

26 Furukawa T, Kanai N, Shiwaku H O, et al. AURKA is one of the downstream targets of MAPK1/ERK2 in pancreatic cancer. Oncogene, 2006, 25: 4831-4839

27 Oktay K, Buyuk E, Oktem O, et al. The c-Jun N-terminal kinase (JNK) functions upstream of Aurora B to promote entry into mitosis. Cell Cycle, 2008, 7: 533-541

Open Access This article is distributed under the terms of the Creative Commons Attribution License which permits any use, distribution, and reproduction in any medium, provided the original author(s) and source are credited. 